\title{
Empleo del catéter doble J para la prevención de las complicaciones urológicas en el trasplante renal
}

\author{
Gonzalo Rodríguez V, Rivero Martínez MD, Trueba Arguiñarena FJ, Martín Martín S, \\ De Castro Olmedo C, Fernández Del Busto E.
}

Hospital Clinico Universitario de Valladolid.

Actas Urol Esp. 2008;32(2):225-229

\section{RESUMEN}

EMPLEO DEL CATÉTER DOBLE J PARA LA PREVENCIÓN DE LAS COMPLICACIONES UROLÓGICAS EN EL TRASPLANTE RENAL

Objetivo: Analizar si la cateterización sistemática de la anastomosis ureterovesical en el trasplante renal mediante un catéter doble $\mathrm{J}$ disminuye la incidencia de las complicaciones urológicas tipo fistula y estenosis ureterales.

Material y métodos: En el periodo comprendido entre agosto de 2003 y diciembre de 2004 realizamos 59 trasplantes renales de donante cadáver. Establecemos dos grupos, grupo A pacientes que durante el trasplante se les coloca un catéter doble J y grupo B pacientes trasplantados sin colocación de catéter. Mediante un estudio retrospectivo analizamos la incidencia de fistulas urinarias, estenosis ureterales y procesos infecciosos en ambos grupos.

Resultados: En el grupo A (con catéter doble J) se detectaron 1 caso de estenosis ureteral y 2 casos (7\%) de fistula urinaria. En un paciente fue necesaria la retirada del catéter al producir obstrucción por coágulos. Trece pacientes (46\%) presentaron un cultivo de orina positivo. En el grupo B (sin catéter doble J) se evidenciaron 4 casos (13\%) de estenosis ureteral y 5 casos (16\%) de fistula urinaria. Nueve pacientes $(29 \%)$ presentaron un cultivo de orina positivo.

Conclusión: El empleo del catéter doble $\mathrm{J}$ de forma profiláctica en el trasplante renal, disminuye las fístulas urinarias y en mayor medida las estenosis ureterales.

Palabras clave: Catéter Doble J. Trasplante renal. Complicaciones urológicas.

\section{ABSTRACT}

THE USE OF DOUBLE J STENT FOR PREVENTION OF UROLOGICAL COMPLICATIONS IN KIDNEY TRANSPLANTS

Objetive: To analyze the impact of the use of the double $\mathrm{J}$ stent in the incidence of urological complications, like fistula urinary and ureteral obstruction in kidney transplants.

Materials and methods: Between August 2003 and December 2005, 59 adult recipients underwent renal transplant. A retrospective study was conducted on two groups of patients: Group A with double $\mathrm{J}$ stent and group B without it. We reviewed the urological complications: fistula, ureteral obstruction and urinary tract infection.

Results: Group A: One ureteral obstruction and two urinary fistulas (7\%) were developed in this group. 13 patients $(46 \%)$ had a positive urinary culture. In only one case was necessary to retire the double $\mathrm{J}$ stent because of ureteral obstruction.

Group B: Four patients (13\%) developed ureteral obstruction and another five (16\%) developed urinary fistula. 9 patients (29\%) had a positive urinary culture.

Conclusion: The routine insertion of a double $\mathrm{J}$ stent in kidney transplants reduces the number of early complications urinary fistula and ureteral obstructions. 
$\mathrm{E}^{\mathrm{n}}$ n los últimos años las complicaciones urológicas del trasplante renal han ido disminuyendo de forma progresiva. En nuestro grupo de trasplante el porcentaje de las mismas se cifra en un $16,6 \%{ }^{1}$. Las alteraciones que se observan tanto en el postoperatorio temprano como en el tardío, fundamentalmente fistulas y estenosis ureterales, se localizan con mayor frecuencia en el tercio distal del uréter.

La cirugía abierta ha dejado paso a las técnicas endourológicas para el tratamiento de estas complicaciones, empleando nefrostomías percutáneas, catéteres doble J, dilataciones con catéter-balón y prótesis metálicas autoexpansibles, obteniéndose muy buenos resultados ${ }^{1}$.

En un intento no solo de tratar estas complicaciones sino también de "prevenirlas" se ha planteado el empleo de forma sistemática y profiláctica del catéter doble $\mathrm{J}$ en el trasplante renal ${ }^{2-9}$.

Sin embargo si revisamos la literatura observaremos posturas muy diversas con respecto a esta medida. Hay quienes consideran que el uso de estos catéteres, dado que no deja de ser un cuerpo extraño, puede aumentar el riesgo de infecciones urinarias ${ }^{2}$, teniendo en cuenta que el paciente trasplantado esta inmunodeprimido, además de los problemas inherentes al uso de estos catéteres como son la migración, la obstrucción de la vía urinaria...etc.

Por ello decidimos realizar un estudio comparativo de manera retrospectiva y mediante un análisis estadístico, entre dos grupos de pacientes sometidos a trasplante renal, un grupo en los colocamos de forma sistemática un con catéter doble J y otro grupo sin él.

\section{MATERIAL Y MÉTODOS}

En el periodo comprendido entre agosto de 2003 y diciembre de 2004 realizamos 59 trasplantes renales de donante cadáver. Establecemos dos grupos, grupo A con catéter doble $\mathrm{J}$ y grupo B sin él. Ambos grupos son comparables en edad, sexo, inmunosupresión empleada y técnica quirúrgica realizada.

\section{Grupo A (Catéter doble J)}

Este grupo esta constituido por 28 pacientes, $9(32 \%)$ de ellos mujeres. La media de edad es de 47.2 años (21-68). El trasplante renal se realizó en la fosa iliaca derecha, salvo en un caso en el que fue implantado en la fosa iliaca izquierda. Para la reimplantación ureteral empleamos la técnica intravesical de Paquin y cateterizamos la anastomosis con un catéter doble J 6 fr. de diámetro y de $20 \mathrm{~cm}$ de longitud.

Los pacientes recibieron tratamiento antibiótico con ciprofloxacino 500 mgr cada $12 \mathrm{~h}$ hasta la retirada del catéter. La terapia inmunosupresora se realizó mediante el empleo de tacrólimus, prednisona y 10 pacientes recibieron además micofenolato de mofetilo. El catéter fue retirado con anestesia local y bajo visión cistoscópica a los 28 dias (4-120) (Tabla 1).

Tabla 1. Características de los pacientes de cada grupo del estudio

\begin{tabular}{|c|c|c|}
\hline & $\begin{array}{c}\text { Grupo A } \\
\text { (catéter JJ) }\end{array}$ & $\begin{array}{c}\text { Grupo B } \\
\text { (sin catéter) }\end{array}$ \\
\hline Mujeres-Hombres & $9-19$ & $13-18$ \\
\hline Edad media & 47,2 & 48,6 \\
\hline Inmunosupresión & $\begin{array}{c}\text { - Tacrólimus } \\
\text { - Prednisona } \\
\text { - Micofenolato } \\
\text { (en } 10 \text { pacientes) }\end{array}$ & $\begin{array}{l}\text { - Tacrólimus } \\
\text { - Prednisona } \\
\text { - Micofenolato } \\
\text { (en } 9 \text { pacientes) }\end{array}$ \\
\hline $\begin{array}{l}\text { Técnica de } \\
\text { reimplantación } \\
\text { ureteral }\end{array}$ & $\begin{array}{c}\text { Técnica } \\
\text { intravesical } \\
\text { de Paquin }\end{array}$ & $\begin{array}{c}\text { Técnica } \\
\text { intravesical } \\
\text { de Paquin }\end{array}$ \\
\hline Catéter doble J & $\begin{array}{l}\text { Con Catéter doble J } \\
(6 \mathrm{fr} ., 20 \mathrm{cms})\end{array}$ & $\begin{array}{l}\text { Sin } \\
\text { catéter }\end{array}$ \\
\hline
\end{tabular}

\section{Grupo B (Sin catéter doble J)}

Este grupo lo forman 31 pacientes, 13 de ellos mujeres (42\%). La media de edad fue de 48,6 años (20-74). Sólo en dos casos el implante fue realizado en la fosa iliaca izquierda. De la misma manera la reimplantación ureteral se llevo a cabo mediante la técnica intravesical de Paquin sin cateterización ureteral. Todos recibieron tratamiento con tacrólimus, prednisona y 9 pacientes además micofenolato de mofetilo.

En el postoperatorio todos los pacientes fueron monitorizados mediante analitica sanguínea (creatinina, urea, hematocrito, leucocitos), cultivo de orina, eco-doppler y gamma- grafia renales (DTPA).

Analizamos el porcentaje de complicaciones aparecidas en ambos grupos especialmente este- 
nosis ureterales, fistulas urinarias y procesos infecciosos, siendo el seguimiento medio de los pacientes de 2 años (1.5-3).

\section{ANALISIS ESTADÍSTICO}

Una vez obtenidos los datos de las distintas complicaciones realizamos un estudio estadístico aplicando el test exacto de Fisher.

\section{RESULTADOS}

Grupo A

En el postoperatorio de este grupo se detectaron 1 caso de estenosis ureteral y 2 casos $(7 \%)$ de fistula urinaria, que clinicamente se manifestaron mediante oligoanuria y deterioro de la función renal.

La estenosis se localizó a nivel de la unión pieloureteral y fue diagnosticada 3 meses después del trasplante renal. Se trató con éxito mediante la colocación de un catéter doble $\mathrm{J}$ durante 6 meses.

El primer caso de fistula urinaria debutó a la semana del trasplante, estando la fuga localizada en el tercio medio del uréter. Inicialmente se trató con técnicas endourológicas no siendo efectivo por lo que se realizó una anastomosis pielopielica. El segundo caso fue diagnosticado un mes después de la cirugía, se localizó a nivel calicial siendo tratada con un catéter doble $\mathrm{J}$ durante 45 dias.

Con respecto a las complicaciones inherentes al empleo de estos catéteres, sólo en un paciente fue necesaria la retirada del mismo, en el $4^{\circ}$ día de postoperatorio, por producir un cuadro obstructivo por coágulos. No observamos ningún caso de migración del catéter.

Dos pacientes $(7 \%)$ presentaron un rechazo agudo del injerto que se resolvió con corticoterapia y 14 pacientes $(50 \%)$ desarrollaron necrosis tubular aguda con retraso en el descenso de los productos nitrogenados.

En cuanto a los procesos infecciosos 13 pacientes $(46 \%)$ presentaron un único cultivo de orina positivo y sólo 6 pacientes $(21 \%)$ presentaron dos cultivos de orina positivos. Las bacterias que fueron aisladas con más frecuencia fueron Pseudomona aeruginosa (31\%), Enterococo faecalis $(26 \%)$ y Escherichia Coli (15\%). Todos los casos se trataron con antibioterapia, solo en un caso fue necesario retirar el catéter doble $\mathrm{J}$ por presentar el paciente una pielonefritis aguda severa.

No se detectó ninguna complicación durante ni tras la retirada de los catéteres doble $\mathrm{J}$, en los pacientes trasplantados.

\section{Grupo B}

En el seguimiento de este grupo se diagnosticaron 4 casos (13\%) de estenosis ureteral y 5 casos $(16 \%)$ de fistula urinaria. Tres de las estenosis fueron diagnosticadas 3 meses después del trasplante. El stop se localizaba en el uréter distal próximo a la reimplantación ureteral. En dos de los casos la alteración se solucionó mediante un catéter doble $\mathrm{J}$ durante una media de 70 días (60-90). En el otro paciente no fue posible la cateterización realizando una dilatación con balón y colocando una prótesis metálica autoexpansible. El cuarto caso de estenosis debutó 1 año después de la cirugía localizándose en el uréter proximal y se trató con técnicas endourológicas inicialmente, no siendo efectivo por lo que se realizó una anastomosis pielopielica.

De las fistulas urinarias observadas en este grupo, un caso se localizó a nivel vesical apareciendo a las 24 horas postrasplante y se corrigió mediante la permanencia de la sonda vesical 60 dias. Los cuatro casos restantes aparecieron dos meses tras la colocación del injerto renal .Dos pacientes presentaron fuga a nivel proximal del uréter y los otros dos a nivel distal. Las estenosis proximales se resolvieron con un catéter doble $\mathrm{J}$ durante 60 días y las distales con la colocación de una prótesis metálica.

En dos pacientes $(6,5 \%)$ se detectó rechazo agudo del injerto que se resolvió con corticoterapia y 12 pacientes (39\%) presentaron necrosis tubular aguda. Además se observó nefrotoxicidad por tacrólimus en un caso que respondió al descenso de la dosis del inmunosupresor.

En el postoperatorio 9 pacientes (29\%) presentaron un cultivo de orina positivo y 2 pacientes $(6,5 \%)$ presentaron dos cultivos de orina positivos. Las bacterias que fueron aisladas con más frecuencia fueron Enterococo faecalis (36\%) y Escherichia Coli (27\%), existiendo un caso de pielonefritis. 
Mediante el análisis de los datos vemos que no existe diferencia entre los procesos de rechazo agudo y necrosis tubular aguda en ambos grupos de pacientes. La incidencia de las complicaciones urológicas (fistula y estenosis ureteral), en el grupo de pacientes en los que se colocó el catéter doble $\mathrm{J}$ fue tres veces menor, aunque la diferencia no fue estadisticamente significativa, aplicando el Test exacto de Fisher

( $\mathrm{P}=0,428$ y $\mathrm{P}=0,356$ respectivamente).

La tasa de cultivos de orina positivos fue mayor en los pacientes trasplantados sometidos a cateterización, siendo el resultado estadisticamente significativo $(P=0,004)$, pero sólo en un paciente tuvo trascendencia clínica desarrollando una pielonefritis aguda. (Tabla 2).

Tabla 2. Complicaciones observadas en los dos grupos de pacientes trasplantados

\begin{tabular}{lccc}
\hline & $\begin{array}{c}\text { Grupo A } \\
\text { (catéter JJ) }\end{array}$ & $\begin{array}{c}\text { GRUPO B } \\
\text { (sin catéter) }\end{array}$ & p \\
\hline Fístula urinaria & $7 \%$ & $16 \%$ & $\mathrm{P}=0,428$ \\
Estenosis ureteral & $3,5 \%$ & $13 \%$ & $\mathrm{P}=0,356$ \\
$\begin{array}{l}\text { Pacientes con cultivo } \\
\text { de orina positivo }\end{array}$ & $67 \%$ & $35 \%$ & $\mathrm{P}=0,004$ \\
\hline
\end{tabular}

\section{DISCUSIÓN}

Las complicaciones urológicas fistulas y estenosis, representan la principal causa de morbimortalidad del enfermo trasplantado ${ }^{1,2}$.

Son debidas a la alteración de la vascularización ureteral que se produce en la manipulación del injerto renal y al realizar la reimplantación ureterovesical. Este compromiso vascular genera isquemia y por tanto necrosis que afecta con mayor frecuencia al tercio distal del uréter ${ }^{1,3,4}$.

Los mecanismos ${ }^{2}$ que se postulan por los que la cateterización ureteral disminuye la incidencia de estas complicaciones son:

- Conseguir una anastomosis ureterovesical más segura y estanca.

- El catéter protege al uréter de compresiones extrinsecas.

- Mantiene una presión intraureteral baja reduciendo el riesgo de fugas.

- Evita los cuadros obstructivos en el postoperatorio inmediato debido a los procesos edematosos en el uréter distal.
Uno de los temas de controversia que se plantea ante el empleo sistemático del catéter en los trasplantes es el riesgo de infecciones, al tratarse de pacientes inmunodeprimidos.

En nuestro estudio, observamos un mayor porcentaje de cultivos positivos en el grupo de pacientes con cateter $\mathrm{J}$, no siendo su repercusión clínica de gran magnitud ya que solamente en un caso fue necesaria la retirada del cateter por pielonefritis severa. En la revisión de la literatura se constata que la mayoria de las complicaciones urinarias por el empleo de catéteres se produce en las cateterizaciones largas ${ }^{8}$, siendo poco frecuentes los problemas cuando el tiempo de cateterización es corto, como es el caso que nos ocupa.

Sin embargo lo que si demuestra nuestra experiencia es que la tasa de fistulas $(7 \%$ en pacientes con cateter frente a $16 \%$ en pacientes sin cateterización) y sobre todo de estenosis ureterales $(3,5 \%$ y $13 \%$ respectivamente) fue significativamente menor en los pacientes trasplantados en los que cateterizamos la reimplantación ureterovesical.

Uno de los objetivos que debemos plantearnos a la hora de realizar un trasplante renal para evitar las complicaciones, es conseguir como se ha comentado con anterioridad una anastomosis ureteral segura y estanca. En los últimos 50 años han sido utilizadas un gran número de técnicas intra y extravesicales para realizar esta anastomosis. La técnica intravesical de PolitanoLeadbetter fue la más empleada a lo largo de los años 60. Hoy en día se emplean técnicas muy diversas (Paquin, Lich-Gregoir, Taguchi, Campos-Freire., etc.) dependiendo en muchas ocasiones de la experiencia del cirujano y de los resultados que haya obtenido en su grupo de trabajo. Existen diversos estudios comparativos sobre las complicaciones urológicas asociadas al empleo de una u otra técnica. Georgiev P. et al. ${ }^{10}$ comparan tres grupos de pacientes trasplantados. En el primer grupo se coloca un catéter doble J sistemáticamente y se realiza una anastomosis extravesical, en los otros dos grupos la colocación del cateter doble $\mathrm{J}$ fue a demanda, en uno de ello se realizó una anastomosis extravesical y en el otro intravesical. La tasa de complicaciones urológicas en cada grupo fue del 5,8\%, $17,9 \%$ y $20,8 \%$ respectivamente. 
Shokier AA. et al. ${ }^{11}$ realizan una comparación entre las técnicas Paquin y Campos-Freire en un total de 400 pacientes sometidos a trasplante renal observando una tasa de complicaciones mucho mayor con la técnica de Paquin $(8,6 \%$ frente a un 4\%) destacando una mayor incidencia de fistulas urinarias. Gurkan et al. ${ }^{12}$ comparan la realización de una uretero-ureterostomía con la técnica extravesical Lich-Gregoir, obteniendo una tasa de complicaciones del 2,9\% y del $8,8 \%$ respectivamente.

Otros estudios ${ }^{13-14}$ demuestran sin embargo una menor tasa de complicaciones con la técnica Lich-Gregoir frente a la anastomosis de Taguchi a pesar de que esta última técnica sea más rápida y sencilla de realizar o la técnica de Shanfield que asocia una mayor incidencia de litiasis vesical y hematuria, que en ocasiones requiere cistoscopia y electrocoagulación endoscópica.

\section{CONCLUSIONES}

La cateterización de la anastomosis ureterovesical mediante el empleo de un cateter doble $\mathrm{J}$ en el trasplante renal, disminuye las fistulas urinarias y sobre todo las estenosis ureterales, no aumentando el riesgo de complicaciones infecciosas ya que el tiempo de permanencia es corto. Tanto la colocación, como la retirada del mismo es un procedimiento sencillo.

Con este estudio, destacamos los buenos resultados que nuestro grupo de trasplante renal ha obtenido mediante el empleo de técnicas endourológicas, tanto para la prevención como para el tratamiento de las fistulas y las estenosis ureterales.

\section{REFERENCIAS}

1. Gonzalo Rodríguez V, Rivero Martínez MD, Trueba Arguiñarena J, Calleja Escudero J, Müller Arteaga C, Fernández del Busto E. Diagnóstico y tratamiento de las complicaciones urológicas del trasplante renal. Actas Urol Esp. 2006; 30(6):619-625.

2. Briones Mardones G, Burgos Revilla FJ, Pascual Santos J, Marcen Letosa R, Pozo Mengual B, Arambarri Segura M, et al. Estudio comparativo entre anastomosis ureteral con y sin cateterización con doble $\mathrm{J}$ en trasplante renal. Actas Urol Esp. 2001;25(7):499-503.
3. Osman Y, Ali-El-Dein B, Shokeir AA, Kamal M, El-Din AB. Routine insertion of ureteral stent in live donor renal transplantation: is it worthwhile?. Adult Urology. 2005;65(5):867871.

4. Pisani F, Iaria G, D'Angelo M, Rascente M, Barletta A, Rizza V, et al. Urologic complications in kidney transplantation. Transplantation Proc. 2005;37(6): 2521-2522.

5. Salahi H, Malek-Hosseini SA, Ghahramani N, Ahmad E, Bahador A, Momtahan S, et al. The efficacy of ureteral stents in prevention of urological complications in renal transplantation. Transplant Proc. 2001;33(5):2668.

6. Moray G, Yagmurdur MC, Sevmis S, Ayvaz I ,Haberal M. Effect of routine insertion of a double-J stent after living related renal transplantation. Transplant Proc. 2005;37 (2):1052-1053.

7. Gerrard ER Jr, Burns JR, Young CJ, Urban DA, Hammontree LN, El-Galley R, et al. Retrograde stenting for obstruction of the renal transplant ureter. Urology. 2005; 66(2):256-260.

8. Salsalone C.V, Maione G, Aseni P, Mangoni I, Soldano S, Minetti E, et al. Advantages of short-time ureteric stenting for prevention of urological complications in kidney transplantation: An 18-year experience. Transplant Proc. 2005; 37(6):2511-2515.

9. Battaglia M, Ditonno P, Selvaggio O, Palazzo S, Bettocchi $\mathrm{C}$, Peschechera R, et al. Double J stent with antireflux device in the prevention of short-term urological complications after cadaveric kidney transplantation: Single-center prospective randomized study. Transplant Proc. 2005;37 (6):2525-2526.

10. Georgiev P, Bóni C, Dahm F, Marcus CF, Wildi S, Rousson $\mathrm{V}$, et al. Routine stenting reduces urologic complications as compared with stenting "on demand" in adult kidney transplantation. Urology. 2007;70(5):893-897.

11. Shokeir AA, Sobh MA, Bakr MA, Ghoneim MA. Vesico-ureteral reimplantation in kidney transplantation from living relative donor: extravesical or transvesical?. Urologic complications and long-term results evaluation. Prog. Urol. 1992; 2(2):241-248.

12. Gurkan A, Yakupoglu YK, Dinckan A, Erdogdu T, Tuncer M, Erdogan O, et al. Comparing two ureter reimplantation techniques in kidney transplant recipients. Transpl Int. 2006;19(10):802-806.

13. Zargar MA, Shahrokh H, Mohammadi Fallah M.R, Zagar H. Comparing Taguchi and anterior Lich-Gregoir ureterovesical reimplantation techniques for kidney transplantation. Transplant Proc. 2005;37,(7):3077-3078.

14. Veale J.L, Yew J, Gjertson DW, Smith CV, Singer JS, Rosenthal JT, et al. Long-term comparative outcomes between 2 common ureteroneocystostomy techniques for renal transplantation. J Urol. 2007;177(2):632-636.

Correspondencia autor: Dra. V. Gonzalo Rodríguez

Servicio de Urología. Hospital Clínico Universitario de Valladolid Ramón y Cajal, s/n - 47005 Valladolid. Tel.: 983420000

E-mail autora: VictoriaCGR@hotmail.com

Información autor: Original - Trasplante renal

Trabajo recibido: octubre 2006

Trabajo aceptado: noviembre 2007 